\title{
BRITISH VIRGIN ISLANDS
}

Area: 130 sq. km

Population: 12,034

Capital: Road Town

Governor: David R. Barwick

Emergency services: 999

The British Virgin Islands comprise over 60 islands and islets (not all inhabited). The largest are Tortola, where the capital is located, and Virgin Gorda.

The Royal Virgin Islands Police Force is very small, with personnel strength of only 65 . In addition to the General Duties Branch, there is a Criminal Investigation Department, to which an internal security Special Branch is attached. Headquarters is at Road Town, Tortola, and there are police stations at East End, West End (both on Tortola) and Virgin Gorda.

Police Headquarters, Station Avenue, Road Town (43422) 